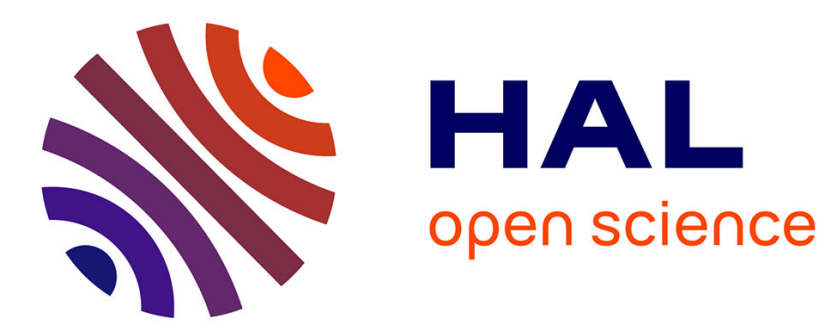

\title{
Autoformation, autonomisation et émancipation
}

Jérôme Eneau

\section{To cite this version:}

Jérôme Eneau. Autoformation, autonomisation et émancipation: De quelques problématiques de recherche en formation d'adultes. Recherches \& éducations, 2016, 16, pp.21-38. hal-01680315

\section{HAL Id: hal-01680315 https://hal.science/hal-01680315}

Submitted on 10 Jan 2018

HAL is a multi-disciplinary open access archive for the deposit and dissemination of scientific research documents, whether they are published or not. The documents may come from teaching and research institutions in France or abroad, or from public or private research centers.
L'archive ouverte pluridisciplinaire HAL, est destinée au dépôt et à la diffusion de documents scientifiques de niveau recherche, publiés ou non, émanant des établissements d'enseignement et de recherche français ou étrangers, des laboratoires publics ou privés. 


\section{Recherches \& éducations}

16 | Octobre 2016

Émancipation et formation de soi Tome 1

\section{Autoformation, autonomisation et émancipation :}

De quelques problématiques de recherche en formation d'adultes

Jérôme Eneau

\section{CpenEdition}

Journals

Édition électronique

URL : http://journals.openedition.org/rechercheseducations/2489

ISSN : $1760-7760$

Éditeur

Société Binet Simon

Édition imprimée

Date de publication : 1 octobre 2016

Pagination : 21-38

ISSN : 1969-0622

Ce document vous est offert par Université Rennes 2

Référence électronique

Jérôme Eneau, «Autoformation, autonomisation et émancipation : », Recherches \& éducations [En ligne], 16 | Octobre 2016, mis en ligne le 30 juin 2017, consulté le 10 janvier 2018. URL : http:// journals.openedition.org/rechercheseducations/2489

Ce document a été généré automatiquement le 10 janvier 2018.

Propriété intellectuelle 


\title{
Autoformation, autonomisation et émancipation :
}

De quelques problématiques de recherche en formation d'adultes

\author{
Jérôme Eneau
}

\section{Introduction}

1 L'émancipation, en formation d'adultes, relève d'un projet ancien dont les racines se retrouvent de manière commune dans de nombreux champs et courants actuels de recherche, tant en France qu'à l'étranger. Au plan étymologique, le terme d'émancipation conserve toujours son sens premier venant du latin emancipare : «se libérer de l'autorité d'autrui » (autorité paternelle, pour l'enfant, autorité morale, pour l'adulte) et par extension, «s'affranchir d'un état d'assujettissement ou de dépendance » (Rey, 1998). En éducation, de manière générale, le terme peut ainsi se résumer, depuis les Lumières, à l'exhortation que Kant a reformulée à propos « d'oser savoir » comme « oser penser par soi-même » (Cornu-Bernot, 2015, p. 90) : "Sapere aude, voilà la devise des Lumières!" (Kant, 1784/2006). Ne plus être sous l'emprise ou la domination d'un autre, oser se servir de son intelligence alors que paradoxalement, " pour être libre, l'homme a besoin d'un maître ", tel est le paradoxe que propose Kant et l'objet même, aujourd'hui encore, de toute philosophie de l'éducation (Cornu-Bernot, 2015).

Or, si du point de vue de l'apprentissage progressif de l'autonomie, pour l'enfant, cette finalité éducative peut aisément être comprise comme une émancipation de l'autorité parentale, le paradoxe d'une autonomie construite dans l'interdépendance, à l'âge adulte, pose toutefois d'autres problèmes. Ce sont ces liens ambigus entre autonomie et émancipation que cet article se propose d'examiner.

Après un bref retour historique sur l'apparition du terme d'émancipation en formation d'adultes, les travaux portant sur l'autoformation permettront de mettre en exergue, dans un premier temps, les liens entre autonomie et émancipation dans des dimensions principalement individuelles, l'apprentissage étant surtout étudié ici sous l'angle de 
processus psychologiques. Nous reviendrons ensuite sur les particularités de la notion d'émancipation, dans les recherches sur l'autoformation, dans une acception plus large et qui renouvelle des questions sociales, sociétales et donc politiques de l'autonomisation. Là, ce sont aussi des dimensions collectives de l'autoformation qui seront prises en compte, puisque ce contexte social est d'abord et avant tout constitué de normes et de valeurs, d'interactions et de négociations. L'émancipation sera alors abordée sous l'angle de ses enjeux théoriques, pour la recherche en formation d'adultes, au prisme de questions à la fois épistémologiques et axiologiques: les liens entre autonomisation et émancipation, de ce point de vue, permettent ainsi de renouer avec une perspective plus «critique » de l'autoformation. Enfin, cet article ouvrira, en guise de conclusion, vers de nouvelles pistes de recherches, grâce aux liens que certains travaux contemporains offrent entre émancipation, empowerment, capacitation et capabilités.

\section{Émancipation et autonomisation : un bref retour historique}

2 À travers l'examen des recherches contemporaines sur la formation et l'autoformation, du côté francophone et du côté anglophone, on retrouve la même aspiration à une double finalité éducative émancipatrice, à la fois individuelle et collective, que porte l'éducation des adultes dans son sens le plus large (Labelle \& Eneau, 2008; Le Boucher, 2015). Cette acception sociale de l'émancipation est toutefois récente, en éducation des adultes, dans la mesure où elle se réfère principalement, dans la constitution du champ, à l'émergence de l'éducation ouvrière et de l'éducation populaire, au tournant des XVIIIe et XIXe siècles, et au croisement d'une triple révolution : humaniste sur le plan des idées, industrielle sur le plan économique et politique sur le plan sociétal. Comme l'ont montré les travaux de Laot et De Lescure (2008), du côté français en particulier, l'histoire de la formation et de l'émancipation des adultes est ainsi ancrée dans cette double dimension individuelle et collective. Elle relève tout à la fois d'une tradition laïque, d'un courant chrétien humaniste mais aussi de l'histoire du mouvement ouvrier qui lui confèrent un double aspect développemental, sur le plan de l'apprentissage individuel, et politique, pour ce qui concerne la prise de conscience collective d'une possible libération d'assujettissement de classes à l'ordre social qui se met alors en place, tout au long du $19^{\text {ème }}$ siècle (Cukier et al., 2013 ; Derycke \& Peroni, 2010 ; Nizet, 2009 ; Vallerie, 2012).

Ce phénomène n'est d'ailleurs pas uniquement français: les travaux francophones et anglophones sur l'éducation des adultes soulignent en effet cet enracinement, pour des raisons relativement similaires dans les sociétés occidentales, au cours des XIXe et XXe siècles: universités populaires, bourses du travail, cercles d'études, community based learning, pédagogies "émancipatrices", de «libération» ou encore de " conscientisation" (liberation pedagogy, emancipatory pedagogy, consciencousness-raising groups, community development) vont fortement influencer cette vision de l'émancipation comme possible libération de différentes formes d'oppression ou de domination, sous l'influence du marxisme et d'idéaux révolutionnaires, en Europe, tout autant que de mouvements civiques, des noirs puis des femmes, aux Etats-Unis ou encore en Amérique du Sud (Carré, 2009 ; Colson, 2005 ; Evans, 2005 ; Lamoureux, 2009 ; Obilade, 2005 ; Tisdell, 2005 ; Wiggins, 2011).

Ainsi, l'éducation des adultes ne fait-elle que renouer, à la fin du XXe siècle, avec l'idéal d'émancipation de la paiedea promue par les Grecs, mais aussi avec une vision idéalisée de 
l'humanisme issue de la Renaissance tout autant qu'avec le projet révolutionnaire formalisé en 1792 par Condorcet (Albero, Gueudet, Eneau \& Blocher, 2015 ; Eneau, à paraitre), autant d'idéaux qui rejoignent une visée d'autonomie personnelle, collective et politique à la fois. L'émancipation, comme l'autonomie, devient alors un concept qui englobe et dépasse la seule perspective d'un apprentissage individuel, fut-il celui d'un "citoyen apprenant ", lui-même "autonome et responsable", une fois devenu adulte, visant à lui permettre d'habiter un monde forgé de manière commune avec d'autres adultes « autonomes, responsables et citoyens» (Labelle, 1996). En d'autres termes, la promotion d'un idéal de liberté, d'égalité et d'accès à la citoyenneté (Lamoureux, 2009) que l'on retrouve, au tournant du XXIe siècle, dans les travaux sur l'autoformation en particulier (Eneau, 2008, 2014).

\section{Autoformation, autonomie et autonomisation}

Les enjeux du développement de l'autonomie de l'apprenant, comme facteur d'émancipation possible à l'âge adulte, sont en effet au cœur de la problématique de l'autoformation, et ce depuis près d'un demi-siècle (Chené, 1983; Tremblay 2003). Ces recherches ont largement souligné les paradoxes et les ambigüités d'une autonomie qui se construit à la fois par soi-même mais aussi par les autres, dans un processus d'interdépendance qui relève d'une autonomisation, comme émancipation, à la fois " procédurale » et « épistémologique », pour reprendre la catégorisation de Candy (1991). Plus encore que pour l'émancipation, les paradoxes du concept d'autonomie, dans les travaux sur l'autoformation, relèvent ainsi d'ambiguïtés fondamentales qui portent tant sur la nature de cette autonomie que sur la valeur qu'on lui accorde dans le processus éducatif. Les confusions restent nombreuses, entre "autonomie de l'apprenant " et " apprentissage autonome », entre " apprenant autonome » et " apprenant susceptible de le devenir ", entre "autonomie fondamentale, autonomie psychologique et autonomie méthodologique » (Chené, 1983). Eminemment autoréférentiel, le concept d'autonomie ne peut que susciter l'ambiguïté, car s'il s'agit pour l'individu autonome de « construire luimême ses propres lois ", l'adulte est toujours confronté aux problèmes des normes extérieures, que celles-ci soient environnementales, institutionnelles ou du fait d'autrui. L'autonomie de l'individu reste donc dans une dialectique entre liberté individuelle et contraintes externes, dans un état intermédiaire, dépendant de la situation et du contexte (Candy, 1991). L'apprentissage de l'adulte, comme l'individu lui-même, ne pourront jamais être qualifiés que de "relativement autonomes». A fortiori, l'émancipation dont il est alors question, dans les travaux sur l'autoformation, relève de multiples dimensions, à la fois épistémologiques, axiologiques et méthodologiques : il peut s'agir en effet, si l'on suit la catégorisation proposée par les travaux sur l'autoformation, d'une autonomisation procédurale (apprendre à " gérer par soi-même » son propre apprentissage), individuelle (apprendre à "se gérer soi-même », sur le plan psychologique, cognitif et émotionnel) et autonomisation collective à la fois (apprendre à vivre de manière "autonome et responsable » dans un monde auquel chacun participe pleinement, en particulier sur le plan de la citoyenneté) (Eneau, 2008). 


\section{Autoformation, autodirection et pouvoir d'agir}

4 Dans un premier sens, le terme d'émancipation est donc proche de celui d'autonomie personnelle, tel que développé à la suite de Kant comme capacité à « penser et agir par soi-même", sans être dépendant de l'enseignement, de la pensée ou de la volonté d'autrui. Jusqu'aux années 1970-1980, cette première signification restera prégnante dans de nombreux courants de recherche et en particulier dans les travaux se réclamant d'une approche « humaniste » de la formation et de l'autoformation.

Souvent assimilée à l'autonomisation comme but de l'autoformation (pour « se passer du maître imposé ", selon l'expression de Dumazedier), cette finalité d'émancipation se retrouve, en France, chez les pionniers de la recherche du domaine (Pineau en particulier) et perdure chez les héritiers de "l'École de Tours" dans une vision existentielle et développementale de la formation des adultes (Eneau, 2008). Dans cette perspective, s'émanciper du pouvoir d'autrui revient à apprendre à maîtriser ou se réapproprier son propre parcours, permet de développer la confiance en soi, la prise de conscience et la reconnaissance des ressources dont chacun dispose, la mobilisation des connaissances des autres et les opportunités qu'offrent le contexte, la situation ou l'environnement, permet de développer des méta-compétences pour apprendre à apprendre, mais permet aussi de transformer le rapport au savoir (Brun, 2001 ; Tremblay, 2003 ; Le Boucher, 2015). La réflexivité et la distance critique sont ici mobilisées dans une perspective "d'actualisation de soi", pour reprendre le vocable des psychologues humanistes; l'émancipation est envisagée dès lors comme un processus d'éducabilité « toujours possible » pour chacun et conserve, dans cette perspective développementale, une forte charge idéologique (Nizet, 2009 ; Tremblay \& Eneau, 2006).

À la même époque, bien que pour d'autres raisons, cette vision se retrouve aussi de manière centrale du côté nord-américain, dans les travaux de Knowles en particulier, s'inscrivant dans la lignée des psychologues humanistes de la «troisième voie » (Maslow, McGregor, Rogers, notamment). Cette perspective humaniste essaimera ensuite aux EtatsUnis et au Canada, où les travaux sur l'andragogy et le self-directed learning iront jusqu'à représenter, selon le mot de Merriam (2001), les deux «piliers» de la recherche du domaine, dans les dernières décennies du XXe siècle. Aujourd'hui, cette vision est notamment présente dans le courant néo-piagétien qui a renouvelé profondément, ces dernières années, l'approche développementale de l'apprentissage adulte, dans les travaux en langue anglaise (Drogo-Severson, 2004 ; Kegan, 1982, 1994, 2000 ; Illeris, 2007 ; Jarvis, 2000 ; Hoare, 2006 ; Merriam, Caffarella \& Baumgartner, 2007 ; Taylor, 2006).

Pour autant, cette vision centrée sur l'individu tend aussi à rabattre les seules capacités d'autoformation (et donc d'autonomisation) à des compétences individuelles, une capacité à diriger et contrôler par soi-même son propre apprentissage (self-directed learning) et donc à un "pouvoir d'agir " (agency) lié à des caractéristiques personnelles, des ressources individuelles, voire à des prédispositions. Les travaux portant sur l'autodirection, l'autodétermination et l'autorégulation, de ce point de vue, restent éminemment centrés sur le "pôle psychologique » de l'autoformation, minorant de fait les dimensions sociales et le rôle du contexte, en particulier, dans lequel l'apprenant « agit » et par lequel il « est agi » (Brookfield, 2005a, 2005b ; Jarvis, 2005 ; Merriam et al., 2007 ; Westwood, 2005 ; Wildermeersch \& Olesen, 2012). 


\section{Autoformation, émancipation et transformation sociale}

5 En parallèle de cette finalité développementale, une autre approche du terme, plus critique et plus radicale, va s'élaborer à partir des années 1980 dans le champ de la recherche; celle-ci repose sur un projet de transformation sociale qui concerne plus particulièrement des groupes sociaux désignés comme "assujettis» ou "opprimés ». Dans la veine des idéologies contestataires des années 1970, de nombreux chercheurs se réclameront ainsi des travaux pionniers de Freire, Illich, Gramsci, Boal, mais aussi de Castoriadis en France ou encore de l'Ecole de Francfort en Allemagne, pour affirmer le droit des individus à plus d'équité et de justice sociale, d'une part, et le rôle fondamental de l'éducation des adultes comme principal vecteur d'émancipation, d'autre part (Castoriadis, 1978/1999 ; Caumières, 2011 ; Freire, 1974, 2006 ; Illich, 1971 ; Lucio-Villegas, 2009 ; Zima, 2005). Pour la formation des adultes et l'autoformation, ces recherches seront principalement développées, à partir des Etats-Unis, grâce aux travaux de Brookfield et de Mezirow en particulier (Brookfield, 2005a, 2005b ; Cranton, 2005 ; Evans, 2005 ; Inglis, 1997 ; Mezirow, 1990, 2001, 2012 ; Mezirow \& Taylor, 2009).

L'éducation des adultes, dans cette "perspective critique " (critical thinking et critical approaches, emancipatory education, transformative perspective et transformative learning, en langue anglaise), vise une triple finalité de liberté, d'égalité et de citoyenneté. Ici, le processus d'apprentissage repose en particulier sur la prise de conscience (conscientization ) des individus vis-à-vis des modalités d'assujettissement auxquels ils sont soumis (frames of reference, meaning schemes) et l'émancipation est alors rapprochée d'autres termes (selfgovernment, empowerment, etc.) pour rappeler la visée politique que contient intrinsèquement la notion dans ses visées d'affranchissement et de libération. Moins présente en France que dans d'autres parties du monde (continent américain, notamment, du Nord comme du Sud), cette perspective va orienter ensuite de nombreux travaux de recherche en formation des adultes, dès la fin des années 1970, et se développer en particulier autour de Jack Mezirow, à la Columbia University de New-York, pour occuper aujourd'hui, et y compris en Europe, une place majeure dans la recherche (Fejes \& Fragoso, 2014 ; Fejes \& Nicoll, 2012).

L'audience restreinte de cette perspective critique, en France, peut s'expliquer pour des questions de traduction et donc d'accessibilité (un seul ouvrage de Mezirow a été traduit, en français, en 2001), mais aussi du fait des controverses suscitées par les modèles de justice sociale et les moyens de remédier aux préjudices vécus par certains groupes sociaux (femmes, minorités ethniques, etc.) que présuppose cette perspective (en terme de discrimination positive, par exemple). Or, tant l'analyse de ces injustices que les moyens d'action, en terme de formation, contreviennent au modèle universaliste français tout autant qu'à la tradition hexagonale de la sociologie critique, héritée notamment de Bourdieu (Eneau, 2008 ; Fraser, 2011 ; Kymlicka, 2003). Ceci n'explique toutefois pas, dans la littérature de langue française sur l'autonomie et l'émancipation, en formation d'adultes, la faible réception des travaux issus de l'Ecole de Francfort, par exemple, alors que ceux-ci ont connu une expansion importante du côté nord-américain, à travers ceux d'Habermas, et alors même que les travaux de Honneth sur la reconnaissance, plus récemment, ont connu quant à eux une diffusion rapide. Ceci n'explique pas non plus, par ailleurs, que la tradition critique et politique de l'autonomie (tout comme la tradition de l'éducation populaire ou de l'autogestion), à la suite des travaux de Castoriadis, pour prendre un autre exemple, ait connu des prolongements nombreux dans les sciences 
sociales (Caumières, 2011 ; David, 2000 ; Tomès \& Caumières, 2011) alors qu'elle n'a que peu (voire pas) de réception en éducation des adultes.

\section{Questions épistémologiques et axiologiques}

6 Quoi qu'il en soit, et alors que ces deux approches "développementale " et « transformatrice » ont contribué à construire historiquement le champ de recherche sur la formation et sur l'autoformation, en se réclamant toutes les deux d'un même ancrage humaniste, sur le plan axiologique, et d'une même perspective " constructiviste ", sur le plan analytique, elles se trouvent de fait relativement éloignées l'une de l'autre, dans le paysage actuel de la recherche en éducation des adultes. Sans opposer l'une à l'autre, la perspective développementale voit ainsi l'émancipation comme un processus "d'autonomisation graduelle», dans les travaux français sur l'autoformation, par exemple, quand la perspective critique vise plutôt à interroger, à la fois comme postulat et comme finalité, l'autonomie de l'adulte comme un "processus de transformation", certes de soi-même comme apprenant, mais aussi et in fine des structures sociales qui contraignent et limitent les possibilités d'autonomisation de chacun tout comme des groupes sociaux concernés. Comme dans les prolongements que rencontre l'expansion du terme empowerment, nous y reviendrons, ces divergences et recompositions ne sont d'ailleurs pas le seul fait de l'autonomisation, comme mode d'émancipation, ni de la seule autoformation, comme approche spécifique de l'éducation des adultes.

\section{L'autonomie comme projet politique d'émancipation}

7 Le premier intérêt consistant à revenir aux proximités entre autonomisation et émancipation, sur le plan épistémologique, vise à réinterroger les finalités du processus d'éducation, entre la prise en compte de l'expérience individuelle et celle du projet politique commun. Suivant Dewey $(1939 / 1976 ; 1938 / 1997)$ ou encore Whitehead (1929/1967), s'il est ainsi possible d'affirmer que l'éducation est liée à «la foi en l'expérience et en la démocratie », selon l'expression de Dewey, la signification accordée par l'individu à sa propre expérience confère à son processus d'apprentissage une valeur particulière : il s'agit non seulement de pouvoir se connaître soi-même, mais aussi de se connaître avec et par les autres, pour penser ensemble le monde commun. L'émancipation est alors proche de l'autonomie et de sa double référence possible à nómos (vómos, la loi) et nomós (vouós, de némô, distribuer, partager), en ce qu'elle permettrait «à la fois de vivre bien soi-même et de bien vivre ensemble » (Freire, 2006). C'est dans cette même acception politique (au sens étymologique) que l'autonomie était déjà utilisée par les Grecs anciens (Castoriadis, 1978/1999 ; Haber, 2009) et que l'autonomisation, comme forme d'émancipation, est liée à l'idée de démocratie; c'est donc dans cette même perspective que l'éducation des adultes comporte d'emblée, aujourd'hui encore, une visée tant morale que politique (Albero et al., 2015 ; Artous, 2010 ; Laugier, 2011; Tremblay, 2009).

On retrouve alors, dans les recherches contemporaines comme dans la tradition de l'éducation populaire (Wiggins, 2011), une visée d'émancipation plaidant contre la " privatisation" des individus et des identités (Inglis, 1997; Jansen \& Wildemeersch ; 1998 ; Mezirow, 1990, 2001, 2012) ou encore, comme chez Freire et Castoriadis, le recours à la praxis comme principal vecteur de transformation, d'autonomisation et 
d'émancipation (Brookfield, 2005c ; Tomès \& Caumières, 2011). Selon Castoriadis, ces principes sont non seulement universalisables mais permettent de rejoindre les niveaux individuels et politiques de l'éducation des adultes, dans un projet reposant sur la démocratie participative, sur la réflexivité, sur une "autolimitation inscrite dans les institutions » et dans un « ethos socialement partagé » (Castoriadis, 1978/1999).

Quoi qu'en s'en différenciant nettement, ce sont d'ailleurs des principes proches que Giddens $(2005,2007)$ propose quant à lui pour penser la «modernité radicale»: autonomie individuelle, réflexivité, transparence, confiance et dialogue représentent en effet pour Giddens « les attributs d'une démocratie moderne ». Celle-ci se construit, selon lui, par une politique "générative", soit la combinaison d'une "politique d'émancipation" (visant à libérer les individus des contraintes qui pèsent sur leur existence) et d'une "politique de vie» (traitant des aspirations des individus eu égard aux questions d'écologie, de travail, de vie de couple ou d'éducation). Ainsi, Giddens (2007) réintègre dans le débat politique la question des «styles de vie » et des «chances de vie » (ou « capabilités »), c'est-à-dire celles des responsabilités, du pouvoir et des choix individuels, tout autant que les responsabilités politiques et collectives d'élaboration d'un environnement permettant à tous, dans des « environnements habilitants ", d'exprimer leur autonomie.

\section{L'émancipation comme « autoformation collective et sociale »}

Mais sur un plan plus pragmatique, ce sont d'autres travaux qui sont actuellement mobilisés pour penser l'émancipation dans ses dimensions opératoires. Ancrée dans la tradition de l'éducation populaire et de l'enseignement mutuel, en France notamment (Labelle, 2008 ; Laot \& de Lescure, 2008) mais aussi et plus largement de l'Ecole Moderne, en Europe, et de l'action communautaire et participative, aux Etats-Unis (Evans, 2005; Obilade, 2005; Tisdell, 2005), cette approche de l'émancipation comme autoformation, non seulement individuelle mais aussi collective et sociale, repose essentiellement sur une pédagogie du «contrat» et du «dialogue " (Eneau, à paraître; Labelle, 1996). En Amérique du Nord, celle-ci est empruntée à Freire, à Bohm ou encore à Bakhtine, dans des méthodes privilégiant l'échange, la conscientisation et la discussion critique, même si, comme le rappelle Lee (2005) à propos des limites de l'approche dialogique, «nous n'utilisons pas le langage : c'est le langage qui nous utilise ».

Popularisée en France par les travaux de Rancière $(1987,2010)$, cette approche dialogique peut être référée à celle de Jacotot et du " Maitre ignorant ", qui pointe tant la nécessaire prise en compte d'une "égalité des intelligences » (ou "égalité des consciences », selon Labelle, 1996) que l'actualité de ces «leçons» de Jacotot pour penser aujourd'hui la "société du mépris» ou encore la lutte contre les inégalités. Les dimensions émancipatrices de la prise en compte des savoirs informels et autodidactiques questionnent d'abord et avant tout, dans ce cadre, les rapports au monde savant et à la culture, et donc à travers les rapports au savoir, les rapports au pouvoir (Derycke \& Peroni, 2010 ; Janvier \& Cukier, 2013).

Cette approche de l'émancipation vise à déconstruire, par le dialogue et l'échange, les processus incorporés de domination tout comme les représentations socialement acceptées. Dans différents dispositifs déployés, tels que ceux d'ATD Quart Monde, des Réseaux d'Echanges Réciproques de Savoirs, de la recherche participative ou encore de l'Education Populaire, et à l'instar de la pédagogie émancipatrice de Freire, il s'agit alors de travailler différents degrés de conscientisation : « prise de conscience individuelle » de 
ses propres savoirs (souvent implicites, souvent expérientiels et non valorisés) ; « prise de conscience collective " (l'individu n'est pas seul à rencontrer un problème, une difficulté, une forme ou une autre de non reconnaissance) ; "prise de conscience sociale » de la manière dont ces savoirs et ces difficultés sont influencés et informés par la société (et donc de l'organisation sociale qui reproduit, consciemment ou non, une valorisation des savoirs et plus largement un ordre social) ; «prise de conscience politique » du passage nécessaire, pour la résolution des problèmes individuels et collectifs, d'une action visant la transformation sociale (Brun, 2001; Hall, 2005; Le Boucher, 2015; Obilade, 2005 ; Vallerie, 2012).

\section{L'émancipation comme « critique sociale »}

9 Ainsi, c'est probablement sur le plan axiologique, voire idéologique, que les notions d'émancipation et d'autonomisation et leurs rapports étroits peuvent être les plus discutés. Leur charge de critique sociale, plus répandue dans la littérature anglo-saxonne que francophone, les rapproche d'autres travaux en philosophie morale et politique, mais aussi et surtout de la philosophie sociale (Boltanski, 2009; Honneth, 2007a, 2007b, 2010 ; Jouan \& Laugier, 2009), dans une dimension étonnamment absente des recherches francophones contemporaines sur l'autoformation.

Dans les recherches sur la formation d'adultes, de manière générale, il semble ainsi tout à fait significatif que la tradition nord-américaine prenne en compte l'antériorité des études multiculturelles ou postcoloniales (cultural studies, African-American studies, etc.), des études féministes (et des gender studies), voire même des théologies de la libération (en Amérique du Sud) ou des pédagogies héritées du maoïsme (en Chine), pour considérer l'éducation des adultes comme un levier majeur d'émancipation possible pour des groupes sociaux discriminés (Brookfield, 2005b ; Cranton, 2005 ; Finger, 2005 ; Mezirow, 2001, 2012 ; Tisdell, 2005 ; Westwood, 2005 ; Wiggins, 2011).

Un autre fait notable, de ce point de vue, est la réappropriation par cette pensée critique américaine d'une tradition française souvent citée (les French studies) revisitant Althusser, Bourdieu, Deleuze ou Derrida, alors qu'en France, les recherches en éducation n'abordent que très peu ces auteurs, si ce n'est parfois à travers la remontée à Foucault et aux liens entre institutions éducatives et pouvoir (Prairat, 2015 ; Vincent, 2015) et donc, à nouveau, aux rapports entre savoir et pouvoir (Aubin, 2009 ; Cukier et al., 2013).

Tout se passe comme si la recherche contemporaine sur l'éducation des adultes et sur l'autoformation, en France, ne s'était pas encore vraiment réappropriée cette dimension critique. C'est plutôt dans des domaines connexes (en philosophie sociale, en sociologie) que les expériences de l'invisibilité et du mépris, de l'aliénation ou de la souffrance au travail, par exemple, font émerger la question de la domination comme une "intériorisation du contrôle ", potentiellement pathogène, et dans les études sur le travail que la question de la critique sociale aborde aujourd'hui de front la reformulation $\mathrm{du}$ « souci de soi » et de ses « dérives » (dans l'exigence permanente de «maximisation de soi », par exemple), à travers lesquelles les sociologues pointent notamment les effets de «domination douce » ou de « despotisme soft » du management contemporain (Boltanski \& Chiapello, 2011 ; Courpasson, 2000 ; Martucelli, 2004 ; Marzano, 2006, 2010).

Il parait alors pour le moins paradoxal que la pensée critique française, bien au delà de la recherche en éducation, de manière générale, et de celle sur la formation des adultes, en particulier, devienne ainsi "institutionnalisée ", comme le souligne Boltanski, voire victime d'une "professionnalisation » de la pensée critique et déconnectée des enjeux 
sociaux actuels (Delmotte \& Lavergne, 2013). Le constat, pour le moins sévère, peut toutefois être relativisé si l'on considère que ce n'est pas tant la notion d'émancipation qui, dans le domaine éducatif, a perdu de son envergure politique, à défaut de sa force de subversion. L'apparition d'un nouveau lexique, comme le reflète le succès des termes $d$ ' empowerment et de " capabilités », en est peut être l'un des principaux symptômes.

\section{Reconfigurations de l'émancipation : entre empowerment et capabilités}

A ce titre, l'émergence de la thématique de l'empowerment n'est pas récente, dans les travaux sur l'autoformation, dans la mesure où les recherches en langue anglaise, dans ce domaine, ont montré depuis de nombreuses années les liens existants entre responsabilité de l'individu et capacité à "diriger par soi-même» son propre apprentissage de manière autonome (Hiemstra, 2005 ; Inglis, 1997). Toutefois, l'ambiguïté est renforcée en français par la difficulté de transposition du terme empowerment, traduit par « autonomisation » (au Québec, en particulier, pour les applications à l'éducation et la gestion), comme « développement de la capacité d'autonomie des personnes » et ce, à la place des termes »empouvoirement», habilitation, appropriation, actualisation, activation ou encore "capacitation" (comme renforcement des capacités), dans une «approche par les capabilités » (Bacqué \& Biewener, 2013; OQLF, 2015; Trépos, 2015 ; Vallerie, 2012).

Dans la littérature managériale, l'empowerment est parfois traduit par «délégation de pouvoir » ou par « responsabilisation des acteurs » mais sa popularité récente, en France tout au moins, lui vient plus probablement de son importation dans le domaine du travail social où, depuis les années 1970, le terme a connu une expansion rapide et continue. Référencée par certains auteurs à une approche «centrée sur le développement du pouvoir d'agir des personnes et des collectivités » (Vallerie, 2012), l'empowerment relève dans ce champ d'une histoire ancienne : lutte de populations discriminées (pour l'égalité des droits civiques) ou encore méthodes, pour l'intervention sociale, de lutte contre l'inégalité sociale, la pauvreté et les discriminations (de race, de genre, de handicap, etc.). Son lien avec l'émancipation, au plan éducatif, lui vient alors de la référence attribuée par les travailleurs sociaux à la démarche de "conscientisation ", empruntée à Freire, tout autant qu'à une possible « transformation collective » des rapports sociaux (Calvès, 2009; Lucio-Villegas, 2009 ; Vallerie, 2012). Dans son sens le plus large, l'empowerment doit alors être travaillé dans une triple dimension: individuelle (renforcement du pouvoir d'agir), collective (interpersonnelle et organisationnelle à la fois) mais aussi politique (et donc sociale) ; autrement dit, l'empowerment travaille les trois dimensions du " pouvoir de », du « pouvoir avec » et du " pouvoir sur » (Bacqué \& Biewener, 2013 ; Jouffray, 2015).

Or, ce que montrent ces évolutions, dans le champ de l'intervention socioéducative, c'est bien que le glissement sémantique de l'émancipation à l'empowerment, la capacitation ou les capabilités s'est accompagné dans les dernières années d'une psychologisation, voire d'une « individualisation de la souffrance " (notamment sociale), venant compenser, en même temps que son expansion dans le registre des pratiques sociales et des luttes contre l'exclusion, un certain désengagement de l'Etat et une injonction au "devoir d'agir " (Bacqué \& Biewener, 2013; Dubois, 2008; Merle \& Antoine, 2015 ; Vallerie, 2012). La capacitation, dans ce sens, renvoie en effet à une « capacité d'agir » que devrait maitriser chacun (y compris les plus vulnérables) pour se « prendre en charge soi-même ». Dans ce 
sens, l'émergence des travaux sur l'empowerment comme ceux sur la capacitation, ainsi que le montre le succès de l'approche de Sen en particulier, peuvent aussi masquer, au delà de l'effacement de la dimension politique de l'émancipation, une réduction des idéaux de l'éducation des adultes à une stricte gestion possible, par chacun, de sa propre " agentivité » (agency). C'est ainsi, notent par exemple Bacqué et Biewener (2013), que s'est développé un certain «individualisme de l'autoréalisation» et que ce qui était jusqu'ici de l'ordre de l'émancipation revient actuellement sous la forme d'une injonction réitérée comme " discipline d'autonomie».

La «domestication» du terme d'empowerment, tout comme celle de l'autonomisation, efface alors l'essence même de sa puissance transformatrice: tandis que l'empowerment relevait à ses débuts d'un idéal de transformation sociale, rappelle Vallerie (2012), la dépolitisation de sa charge critique ressort aujourd'hui d'une instrumentalisation de l'intervention et ne risque bien, au contraire, que de rabattre la relation éducative à une logique de «transaction commerciale » plus qu'émancipatrice, et d'engendrer in fine des « effets iatrogènes » ... le remède tuant le patient.

Pour l'autoformation, il est peut être temps alors de repenser la force émancipatrice de ces notions d'empowerment et d'autonomisation pour retrouver, au delà des évolutions épistémiques et des glissements sémantiques, l'ambition d'affranchissement de la doxa et remonter ainsi à l'exhortation de Kant à " penser par soi-même ». Il ne s'agit pas tant de revenir ici à un combat passéiste que de retrouver la puissance évocatrice des liens entre autonomie individuelle et autonomie collective, comme le pensait Castoriadis, ou encore entre éducation et démocratie, comme le proposait Dewey. Et alors qu'en des temps troubles, les limites entre philosophie de l'action et idéologie de la mobilisation restent une possibilité utile à envisager, peut être faut-il rappeler, à l'instar d'Alinsky, que comme le pouvoir, le savoir ne se donne pas : il s'acquiert collectivement (Bacqué \& Biewener, 2013).

\section{BIBLIOGRAPHIE}

Albero, B., Gueudet, G., Eneau, J., \& Blocher, J-N. (2015). Formes d'éducation et processus

d'émancipation. Rennes : Presses Universitaires de Rennes.

Artous, A. (2010). Démocratie, citoyenneté, émancipation. Paris : Syllepse.

Aubin, F. (2009). Intellectuels et émancipation. Entre compétence technique et conviction éthique. In G. Tremblay (dir.), L'émancipation, hier et aujourd'hui. Perspectives françaises et québécoises (pp. 249-258). Québec: Presses de l'Université du Québec.

Bacqué, M.H., \& Biewener, C. (dir.) (2013). L'empowerment, une pratique émancipatrice. Paris : La Découverte (Collection Politique et Société).

Boltanski, L. (2009). De la critique. Précis de sociologie de l'émancipation. Paris : Gallimard.

Boltanski, L. \& Chiapello, E. (2011). Le nouvel esprit du capitalisme. Paris: Gallimard. 
Brookfield, S. (2005a). Adult learning: an overview. In A. C. Tuijnman, (dir.). International encyclopedia of adult education and training (pp. 375-380). New-York: Pergamon-Elsevier.

Brookfield, S. (2005b). Critical Thinking. In L. M. English (dir.). International Encyclopedia of Adult Education (pp. 168-173). New-York: Palgrave Macmillan.

Brookfield, S. (2005c). Praxis. In L. M. English (dir.). International Encyclopedia of Adult Education (pp. 504-508). New-York : Palgrave Macmillan.

Brun, P. (2001). Emancipation et connaissance. Les Histoires de vie en collectivité. Paris : L'Harmattan.

Calvès, A. E. (2009). "Empowerment" : généalogie d'un concept-clé du discours contemporain sur le développement. Revue Tiers Monde, 4 (200), 735- 749.

Candy, P. (1991) Self-direction for lifelong learning. A comprehensive guide to theory and practice. San Francisco: Jossey Bass.

Carré, D. (2009). De l'émancipation éducative à l'émancipation éducationnelle ? In G. Tremblay (dir.), L'émancipation, hier et aujourd'hui. Perspectives françaises et québécoises (pp. 259-268). Québec : Presses de l'Université du Québec.

Castoriadis, C. (1978/1999). Les Carrefours du labyrinthe (6 tomes). Paris : Seuil/Points.

Caumières, P. (2011). Castoriadis : critique sociale et émancipation. Paris : Textuel.

Chené, A. (1983). The concept of autonomy in adult education: a philosophical discussion. Adult Education Quarterly, 34(1), 38-47.

Colson, D. (2010). Eclectisme et dimension autodidactique de l'anarchisme ouvrier. In M. Derycke \& M. Peroni (dir.). Figures du maître ignorant. Savoir et émancipation (pp. 371-405). Saint-Etienne : Publications de l'Université de Saint-Etienne.

Cornu-Bernot, L. (2015). Transformer les rapports du sujet avec le monde. In B. Albero, G. Gueudet, J. Eneau \& J-N. Blocher (2015). Formes d'éducation et processus d'émancipation (pp. 89-95). Rennes : PUR.

Courpasson, D. (2000). L'action contrainte. Organisations libérales et domination. Paris: PUF.

Cranton, P. (2005). Transformative learning. In L. M. English (dir.). International Encyclopedia of Adult Education (pp. 630-637). New-York: Palgrave Macmillan.

Cukier, A. (2013). Travail, critique et émancipation : l'enjeu du pouvoir. In A. Cukier, F. Delmotte, \& C. Lavergne (dir.). Emancipations, les métamorphoses de la critique sociale (pp. 187-227).

Bellecombes-en-Bauges : Editions du croquant.

Cukier, A., Delmotte, F., Lavergne, C. (dir.) (2013). Emancipation, les métamorphoses de la critique sociale. Paris : Editions du croquant.

David, G. (2000). Cornelius Castoriadis. Le projet d'autonomie. Paris : Editions Michalon.

Delmotte, F. \& Lavergne, C. (2013). De la sociologie de la critique aux impasses actuelles de la critique sociale. Entretien avec Luc Boltanski. In A. Cukier, F. Delmotte, \& C. Lavergne (dir.). Emancipations, les métamorphoses de la critique sociale (pp. 31-60). Bellecombes-en-Bauges : Editions du croquant.

Derycke, M., \& Peroni, M. (dir.) (2010). Figures du maître ignorant. Savoir et émancipation. SaintEtienne : Publications de l'Université de Saint-Etienne.

Dewey, J. (1938/1997). Experience and education. New York: First Touchstone Edition. 
Dewey, J. (1939/1976). Creative democracy: The task before us. In J. Boydston (Ed.). John Dewey: The later works, 1925-1953. vol. 14 (pp. 224-230). Carbondale: Southern Illinois University Press. Drago-Severson, E. (2004). Becoming adult learners. Principles and practices for effective development. NYC : Teachers College Press - Columbia University.

Dubois J.-L. (2008). Repenser l'action collective : une approche par les capabilités. Paris: l'Harmattan.

Eneau, J. (2008). From autonomy to reciprocity, or vice-versa? French Personalism's contribution to a new perspective on self-directed learning. Adult Education Quarterly vol. 58, n 3, 229-248.

Eneau, J. (2014). Emancipation. In A. Jorro (dir.). Dictionnaire des concepts de la professionnalisation (pp. 91-94). Bruxelles: De Boeck.

Eneau, J. (à paraitre). From self-directed learning to self-formation: transforming the self through Bildung. In A. Laros, T. Fuhr \& E. Taylor (Eds). Transformative Learning meets Bildung. Rotterdam (NL): Sense Publishers.

Evans, D. R. (2005). Popular education and conscientization. In A. C. Tuijnman, (dir.). International encyclopedia of adult education and training (pp. 89-92). New-York: Pergamon-Elsevier.

Fejes, A. \& Fragoso, A. (2014). Mapping power in adult education and learning. European Journal for Research on the Education and Learning of Adults (Rela), 5(1), 7-11.

Fejes, A. \& Nicoll, K. (2012). Approaches to research in the education and learning of adults. European Journal for Research on the Education and Learning of Adults (Rela), 4(1), 7-16.

Finger, M. (2005). Critical Theory. In L. M. English (dir.). International Encyclopedia of Adult Education (pp. 165-168). New-York : Palgrave Macmillan.

Fraser N. (2011). Qu'est-ce la justice sociale? Reconnaissance et redistribution. Paris : La Découverte. Freire, P. (1974). Pédagogie des opprimés, suivi de Conscientisation et révolution, Paris : Maspero. Freire, P. (2006). Pédagogie de l'autonomie. Savoirs nécessaires à la pratique pédagogique. Toulouse : Erès.

Giddens, A. (2005). La constitution de la société. Eléments de la théorie de la structuration. Paris : PUF (Quadrige).

Giddens, A. (2007). Les conséquences de la modernité. Paris : L'Harmattan.

Haber, S. (2009). L'autonomie sociale comme forme d'action. Le paradigme du travail. In M. Jouan \& S. Laugier (dir). Comment penser l'autonomie? Entre compétences et dépendances (pp. 269-291). Paris: PUF.

Hall, B. L. (2005). Participatory research. In A. C. Tuijnman, (dir.). International encyclopedia of adult education and training (pp. 187-194). New-York: Pergamon-Elsevier.

Hiemstra, R. (2005). Self-directed learning. In A. C. Tuijnman, (dir.). International encyclopedia of adult education and training (pp. 427-433). New-York: Pergamon-Elsevier.

Hoare, C. (2006). Handbook of adult development and learning. NYC: Oxford University Press.

Honneth, A. (2007a). La lutte pour la reconnaissance (1 ${ }^{\text {ère }}$ édition 1992). Paris : Cerf.

Honneth, A. (2007b). La réification. Petit traité de théorie critique. Paris : Gallimard.

Honneth, A. (2010). La société du mépris. Paris : La Découverte.

Illeris, K. (2007). How we learn. London : Routlege.

Illich, I. (1971). Une société sans école. Paris : Seuil. 
Inglis, T. (1997). Empowerment and emancipation. Adult Education Quarterly 48(1), 3-17.

Jansen, T. \& Wildemeersch, D. (1998). Beyond the myth of self-actualization: reinventing the community perspective of adult education. Adult Education Quarterly 48(4), 216-226.

Janvier, A. \& Cukier, A. (2013). La question politique de l'émancipation. Entretien avec Jacques Rancière. In A. Cukier, F. Delmotte, \& C. Lavergne (dir.). Emancipations, les métamorphoses de la critique sociale (pp. 137-160). Bellecombes-en-Bauges : Editions du croquant.

Jarvis, P. (2000). Learning to be a person in society. London: Routledge.

Jarvis, P. (2005). Sociology of adult learning. In A. C. Tuijnman, (dir.). International encyclopedia of adult education and training (pp. 158-163). New-York: Pergamon-Elsevier.

Jouan, M., \& Laugier, S. (2009). Comment penser l'autonomie? Entre compétences et dépendances. Paris : PUF.

Jouffray, C. (2015). Passer des discours sur le pouvoir d'agir au pouvoir d'agir en action : une condition pour transformer les pratiques et les logiques à l'œuvre. Sciences et actions sociales, $\mathrm{n}^{\circ} 2$. Kant, E. (1784/2006). Qu'est ce que les Lumières ? réédition Kant E. \& Mendelssohn, M. (2006). Paris: Mille et une nuits.

Kegan, R. (1982). The evolving self. Problem and process in human development. Cambridge (MA). Harvard University Press.

Kegan, R. (1994). In and over our heads. The mental demands of modern life. Cambridge (MA): Harvard University Press.

Kegan, R. (2000). What "form" transforms? A constructive-developemental approach to Transformative learning. In J. Mezirow (and ass.) (2000). Learning as transformation. Critical perspectives on a theory in progress (pp. 35-69). San Francisco (CA) : Jossey Bass.

Kymlicka, W. (2003). Les théories de la justice. Une introduction. Paris : La Découverte (Poche).

Labelle, J. M. (1996). La réciprocité éducative. Paris : PUF.

Labelle, J. M. (2008). S'éduquer dans les cours d'adultes, en France, au dix-neuvième siècle. In J.M. Labelle \& J. Eneau (dir.). Apprentissages pluriels des adultes : questions d'hier et d'aujourd'hui (pp. 31-43). Paris : L'Harmattan.

Labelle, J.M. \& Eneau, J. (dir.) (2008). Apprentissages pluriels des adultes : questions d'hier et d'aujourd'hui. Paris : L'Harmattan.

Lamoureux, D. (2009). Féminisme et mouvement des femmes. Entre émancipation et libération. In G. Tremblay (dir.), L'émancipation, hier et aujourd'hui. Perspectives françaises et québécoises (pp. 47-61). Québec : Presses de l'Université du Québec.

Laot, F., \& De Lescure, E. (dir.) (2008). Pour une histoire de la formation, Paris : L'Harmattan.

Laugier, S. (2011). L'éducation des adultes comme philosophie morale. Éducation et didactique, 5(3), 55-64.

Le Boucher, C. (2015). Facteurs de pérennisation d'un dispositif de formation par les pairs. Le cas des Réseaux Réciproques d'Echanges de Savoirs. Thèse de Doctorat en Sciences de l'Education. Rennes : Université Rennes 2.

Lee, M. (2005). Dialogue. In L. M. English (dir.). International Encyclopedia of Adult Education (pp. 185-189). New-York: Palgrave Macmillan. 
Lucio-Villegas, E. (2009). Paulo Freire. In J.M. Barbier, E. Bourgeois, G. Chapelle \& J.C. RuanoBobalan (dir.). Encyclopédie de la formation, (pp. 970-971). Paris : PUF.

Martuccelli, D. (2004). Figures de la domination. Revue française de sociologie 45(3), 469-497.

Marzano, M. (2006). Je consens donc je suis ... Ethique de l'autonomie. Paris : PUF.

Marzano, M. (2010). Extension du domaine de la manipulation. Paris : Hachette.

Merle, P., \& Antoine, J. (dir.) (2015). L'empowerment à la française. Empowerment, développement du pouvoir d'agir, du faire participer, de la citoyenneté : quelles relances pour quel travail social ? Forum - Revue de la Recherche en sciences sociales, $n^{\circ}$ 144-145.

Merriam, S. (2001). Andragogy and self-directed learning: pillars of adult learning theory. New directions for adult and continuing education, $\mathrm{n}^{\circ}$ 89, 3-13.

Merriam, S., Caffarella, R. \& Baumgartner, L. (2007). Learning in adulthood. A comprehensive guide. San Francisco: Jossey-Bass.

Mezirow, J. (dir.) (1990). Fostering critical reflection in adulthood. A guide to transformative and emancipatory learning. San Francisco : Jossey Bass.

Mezirow, J. (2001). Penser son expérience. Développer l'autoformation. Lyon: Chronique Sociale.

Mezirow, J. (2012). Learning to think like an adult. Core concepts of Transformation Theory. In E.W. Taylor \& P. Cranton (and ass.). The handbook of Transformative Learning. Theory, research, and practice (pp. 73-95). San Francisco (CA): Jossey-Bass.

Mezirow, J., \& Taylor, E.W. (2009). Transformative learning in practice. Insights form community, workplace and higher education. San Francisco (CA) : Jossey Bass.

Nizet, J. (2009). Les évolutions idéologiques dans le domaine de la formation des adultes. In J.M. Barbier, E. Bourgeois, G. Chapelle \& J.C. Ruano-Bobalan (dir.). Encyclopédie de la formation, (pp. 967-996). Paris: PUF.

Obilade, O. (2005). Participatory Action Research. In L.M. English (dir.). International Encyclopedia of Adult Education (pp. 460-465). New-York: Palgrave Macmillan.

OQLF (2015). Office Québécois de la Langue Française (OQLF) - Le grand dictionnaire terminologique (en ligne : http://gdt.oqlf.gouv.qc.ca/, consulté le 03.12.2015).

Prairat, E. (2015). Hétérotopies et conceptions normatives. In B. Albero, G. Gueudet, J. Eneau \& JN. Blocher (2015). Formes d'éducation et processus d'émancipation (pp. 57-68). Rennes : PUR.

Rancière, J. (1987) Le Maitre ignorant. Cinq leçons sur l'émancipation intellectuelle. Paris : Fayard.

Rancière, J. (2010). Choses (re)dites. In M. Derycke \& M. Peroni (dir.). Figures du maître ignorant. Savoir et émancipation (pp. 408-427). Saint-Etienne : Publications de l'Université de Saint-Etienne.

Rey, A. (1998). Emancipation. Dictionnaire historique de la langue française (pp. 1211-1212). Paris: Editions Le Robert.

Taylor, K. (2006). Autonomy and self-directed learning: a developmental journey. In C. Hoare (ed.). Handbook of adult development and learning (pp. 196-218). New York (NY): Oxford University Press.

Tisdell, E.J. (2005). Emancipatory education. In L. M. English (dir.). International Encyclopedia of Adult Education (pp. 205-210). New-York : Palgrave Macmillan.

Tomès, A, \& Comières, P. (2011). Cornelius Castoriadis. Réinventer la politique après Marx. Paris : PUF.

Tremblay, G. (dir.) (2009), L'émancipation, hier et aujourd'hui. Perspectives françaises et québécoises. Québec : Presses de l'Université du Québec. 
Tremblay, N. A., \& Eneau, J. (2006). Sujet, société et autoformation. Regards croisés du Québec et de France. Education Permanente ${ }^{\circ}$ 168, 75-88.

Tremblay, N.A. (2003). L'autoformation. Pour apprendre autrement. Montréal : Presses de l'Université de Montréal.

Trépos, J.Y. (2015). L'empowerment, entre puissance et impuissances. Sciences et actions sociales, $n$ ${ }^{\circ} 2$.

Vallerie, B. (dir.) (2012). Interventions sociales et empowerment. Développement du pouvoir d'agir. Paris : L'Harmattan.

Vincent, H. (2015). Foucault éducateur : un art de l'écriture et un modèle d'autoformation. Le Télémaque 2015/1, n 47, 71-86.

Vincent, H. (dir.) (2015). Dossier - Michel Foucault : héritages et perspectives en éducation et formation. Le Télémaque 2015/1, nº 47.

Westwood, S. (2005). Critical approaches to adult education. In A. C. Tuijnman, (dir.). International encyclopedia of adult education and training (pp. 61-65). New-York: Pergamon-Elsevier.

Whitehead, A. N. (1929/1967). The aims of education. New York: Free Press.

Wiggins, N. (2011). Critical pedagogy and popular education: towards a unity of theory and practice. Studies in the Education of Adults vol 43, $\mathrm{n}^{\circ} 1,34-49$.

Wildemeersch, D., \& Olesen, H.S. (2012). The effects of policies for the education and learning of adults - from 'adult education' to 'lifelong learning', from 'emancipation' to 'empowerment'. European Journal for Research on the Education and Learning of Adults, 3(2), 97-101.

Zima, P.V. (2005). L'Ecole de Francfort. Dialectique de la particularité (1 ère édition 1974). Paris : L'Harmattan.

\section{RÉSUMÉS}

Les recherches sur l'autoformation, en français comme en anglais, ont développé un champ conceptuel riche où les notions d'autonomie et d'émancipation sont parfois confondues, dans leur acception originelle, même si l'histoire des différents courants de recherche a ensuite emprunté différentes voies d'interprétation. Ces recherches ont ainsi abordé des dimensions tant psychologiques que sociales, axiologiques et même politiques de l'autonomisation et de l'émancipation. A travers un triple examen étymologique, historique et théorique des travaux sur l'autoformation, cet article propose de dégager les principales lignes de tension, dans la recherche du domaine, permettant d'articuler ces notions. La discussion finale ouvre sur les prolongements actuels de ces questions autour de l'emploi des termes d'empowerment, de capacitation et de capabilités, qui renouvellent les rapports et les enjeux des conditions et finalités même de l'autoformation, si ce n'est de l'éducation des adultes, de manière plus générale.

Research on self-education and self-directed learning, respectively in French and English works, has enriched the conceptual field in adult education where the notions of autonomy and emancipation are sometimes confused in their original meaning, despite different paths of research and different ways of interpretation. The research thus addressed psychological, social, axiological and even political aspects of autonomy and emancipation. This article, through a triple etymological, historical and theoretical examination of the work in this field, proposes to identify the main lines of tension for articulating these differents notions. Finally, the discussion 
opens on current projections about the use of terms like empowerment, capacitation and capabilities, which renew issues for self-education and self-directed learning, if not more generally for adult education.

INDEX

Keywords : adult education, autonomization, self-education, self-directed learning, emancipation

Mots-clés : formation d'adultes, autonomisation, autoformation, émancipation

\section{AUTEUR}

JÉRÔME ENEAU

CREAD - Université Rennes 2 